\title{
7 Intensivmedizinische Maßnahmen zur Aufrechterhaltung der Organfunktionen
}

\author{
Sven-Olaf Kuhn und Klaus Hahnenkamp
}

Die Kenntnis der komplexen, neuroendokrin vermittelten Veränderungen im Rahmen des irreversiblen Hirnfunktionsausfalls (IHA), insbesondere der Hämodynamik und des Elektrolytund Flüssigkeitsgleichgewichtes, ist Voraussetzung für ein effektives und proaktives DonorManagement. Die intensivmedizinische Versorgung des potenziellen Organspenders unterscheidet sich prinzipiell nicht von den üblichen intensivmedizinischen Maßnahmen, beinhaltet aber vor allem mit der Hormonersatztherapie einige Besonderheiten und hat direkte Auswirkung auf den Transplantationserfolg.

Die qualifizierte intensivmedizinische Spenderkonditionierung, wenn erforderlich auch durch eine Therapieeskalation, ist neben der verbesserten Transplantatkonservierung und der Organentnahmen bei sogenannten „expanded criteria donors" eine Voraussetzung für ein optimales Transplantationsergebnis und in erster Linie Aufgabe des Intensivmediziners (Rahmel 2019).

\subsection{Pathophysiologische Veränderungen durch irreversiblen Hirnfunktionsausfall}

Durch die Beeinträchtigung oder den Verlust zentraler, hormoneller Regulationsmechanismen beim Ausfall der Hirnfunktionen (z.B. dem Untergang der Vaguskerne) kommt es vor allem zu Störungen der endokrin vermittelten Regulation mit schweren hämodynamischen und pulmonalen Veränderun- 
gen sowie systemischer Inflammation (Poppelaars u. Seelen 2016; Amado et al. 1995). Sehr häufig sind Hypothermie, Hypotonie und ein zentraler Diabetes insipidus. Durch die Freisetzung von proinflammatorischen Mediatoren, aktivierten Enzymsystemen und freien Sauerstoffradikalen kann es außerdem zur Gefäßendothelschädigung, Aktivierung des Gerinnungs- und Komplementsystems und einer systemischen Entzündungsreaktion ähnlich eines systemic inflammatory response syndrome (SIRS) kommen.

\subsection{Intensivmedizinische Maßnahmen beim potenziellen Organspender}

Die am 01.09.2020 in Kraft getretene Richtlinienänderung der Bundesärztekammer zur Spendererkennung unterstreicht, dass die Aufrechterhaltung der Organfunktionen bis zur Feststellung des bekundeten oder mutmaßlichen Willens notwendig ist, um eine gewollte Organspende ermöglichen zu können. Das TPG legt weder den Behandlungsbedarf noch den Zeitrahmen dafür fest. Es heißt in der Richtlinienänderung weiter,

„solange der irreversible Hirnfunktionsausfall noch nicht festgestellt ist, sollte mit dem Patientenvertreter geklärt werden, ob Maßnahmen zur Wiederherstellung der Herz-Kreislauffunktion dem Patientenwillen entsprechen. Dabei muss zwischen einem Organspendewunsch und einem Willen zur Therapiebegrenzung abgewogen werden" (Bundesärztekammer 2020).

Der Umfang der intensivmedizinischen Maßnahmen zur Aufrechterhaltung der Organfunktionen muss mit den nächsten Angehörigen kommuniziert und abgestimmt werden. Das schließt erweiterte Maßnahmen zur Aufrechterhaltung der Organfunktion (z.B. Maßnahmen zur Wiederherstellung der HerzKreislauf-Funktion wie Herzdruckmassage oder Organersatzverfahren) ein.

Die Therapie eines potenziellen Organspenders beginnt strenggenommen bereits mit der Diagnose einer schweren Hirnschädigung. Kommt es trotz aller Bemühungen zum IHA, müssen die intensivmedizinischen Maßnahmen vollumfänglich fortgeführt werden. Ein proaktives Donor-Management zur Behandlung der oft foudroyant verlaufenden hämodynamischen Veränderungen und Flüssigkeits- und Elektrolytverschiebungen verbessert die Qualität der transplantablen Organe und damit den Erfolg der Transplantation (Angel et al. 2006; Kotloff et al. 2015; Malinoski et al. 2013; Patel et al. 2017). Die einzelnen Maßnahmen zur Spenderkonditionierung orientieren sich prinzipiell an den allgemein gültigen Therapieprinzipien zur Aufrechterhaltung der Homöostase und dem Organsupport kritisch Kranker (Citerio et al. 2016). Die Zielparameter sind in Kapitel 7.7.6 zusammengefasst. 


\section{Die Qualität der intensivmedizinschen Maßnahmen hat direkten Einfluss auf den Erfolg einer Transplantation. Die Behandlung des potenziellen Organspenders beginnt bereits mit dessen Identi- fizierung.}

\subsection{Hämodynamik}

Die infolge der rostro-caudalen Hirnstammeinklemmung auftretenden Sympathikus- und Parasympathikusaktivierung auf Höhe der Pons resultiert in einer ausgeprägten Hypertonie und Bradykardie (Cushing-Reflex) gefolgt von einer durch die Ischämie im Bereich der Medulla oblongata überschießenden Katecholaminfreisetzung, dem sogenannten Katecholaminsturm („autonomic storm“). Vor allem die exzessive adrenerge Kreislaufdysregulation führt bei bereits bestehender a-Rezeptor-vermittelter Vasokonstriktion mit erhöhtem pulmonal- und systemisch-vaskulärem Widerstand durch die Steigerung der Kontraktilität und Herzfrequenz zu einer Zunahme des HerzZeit-Volumens. Die Erhöhung der Nachlast kann konsekutiv zum Linksherzversagen (neurogenes Lungenödem), ausgeprägte Vasospasmen zur Myokardischämie und ventrikulärer Dysfunktion führen. Diese sind prinzipiell reversibel (Madan et al. 2017; Martin-Loeches et al. 2019; Nasr et al. 2017). Im weiteren Verlauf der Hirnstammeinklemmung und nach vollständigem Ausfall aller Hirnfunktionen bewirken die wieder sinkenden Katecholaminspiegel einen Abfall des peripheren Gefäßwiderstandes mit ausgeprägter Hypotonie. Zur differenzierten Volumen- und Katecholamintherapie sollte deshalb großzügig ein erweitertes hämodynamisches Monitoring verwendet werden. Kontinuierliche Verfahren wie die Pulskonturanalyse sind ebenso geeignet wie serielle Echokardiografien (Bednarczyk et al. 2017; Kotloff et al. 2015).

Zur Behandlung des Hypertonus werden bevorzugt kurzwirksame Antihypertensiva (z.B. Urapidil) eingesetzt, bei Hypotonie und ausgeglichenem Volumenstatus vorrangig Katecholamine und Vasopressin. Während Dopamin und Noradrenalin in Deutschland lange die Vasopressoren der Wahl waren, unterstützen aktuellere Daten die Gabe des Polypeptid-Hormons ArgininVasopressin (AVP) (Plurad et al. 2012). Dessen gefäßtonisierende Wirkung resultiert aus der Bindung an die V1-Rezeptoren der Gefäßmuskulatur. Dobutamin ist zur Inotropie- und Chronotropiesteigerung geeignet.

Eine aktuelle Untersuchung legt nahe, dass nach Eintritt des irreversiblen Hirnfunktionsausfalls der Sauerstoffkonsum der Leber zu- und die Nierendurchblutung abnimmt. Die Studienergebnisse unterstreichen die Notwen- 
digkeit einer organspezifischen Bewertung und Optimierung der Transplantatqualität vor der Transplantation, der Optimierung der metabolischen Leberbedingungen und zur Verbesserung der Nierenperfusion bei gleichzeitiger Unterstützung der zellulären Entgiftung (Van Erp et al. 2018a). Die Dauer der kalten Ischämie ist mit einer höheren Rate von Transplantatdysfunktionen insbesondere bei älteren postmortalen Nierenspenden korreliert. Dopamin besitzt anti-oxidative Eigenschaften. Durch seine Fettlöslichkeit und damit gute Diffusion nach intrazellulär wurde ein protektiver Effekt gegen eine Kälte-Ischämie und Reperfusionsschaden sowie eine anti-inflammatorische Wirkung auf das Transplantat beschrieben (Benck et al. 2018; Birtan et al. 2018; Schnuelle et al. 2019; Schnuelle et al. 2017). Außerdem wurde ein verbessertes Überleben nach Herztransplantationen durch Verringerung des akuten Rechtsherzversagens berichtet (Benck et al. 2011). Die DSO und die Deutsche Transplantationsgesellschaft (DTG) empfehlen präoperativ die Gabe von Dopamin in einer Dosierung bis $4 \mu \mathrm{g} /$ $\mathrm{kg} / \mathrm{min}$ für mindestens $6 \mathrm{~h}$ vor Organentnahme (DSO 2016 und s. Tab. 3 in Kap. 7.7.6).

Die konsequente hämodynamische Stabilisierung des Organspenders nach Feststellung des IHA ist mit einer verbesserten Transplantatqualität verbunden. Die „relax and repair"- bzw. die „wait, treat and see"-Strategie hat zu einer Steigerung der Zahl und der Qualität bei Nieren- und Herztransplantationen geführt (Borbely et al. 2015; Martin-Loeches et al. 2019). Es wird ein mittlerer arterieller Druck > $65 \mathrm{mmHg}$ bzw. eine linksventrikuläre Ejektionsfraktion (LVEF) von mindestens 45\% zur Sicherstellung einer adäquaten Organperfusion angestrebt (s. Kap. 7.7.6).

\subsection{Flüssigkeitstherapie, Hämotherapie}

Der Flüssigkeitsverlust infolge des Diabetes insipidus centralis bzw. eine relative Hypovolämie durch den Verlust des Sympathikotonus muss rasch korrigiert werden. Zum Erhalt eines ausreichenden Perfusionsdrucks und einer Stundendiurese von mindestens $1 \mathrm{ml} / \mathrm{kg} / \mathrm{h}$ werden vorrangig kristalloide Vollelektrolytlösungen infundiert, bei ausgeprägter Hypernatriämie und Hypovolämie sind Halbelektrolytlösungen oder 5\% Glucoselösungen geeignet. Vor allem im Hinblick auf eine Lungenspende ist aber eher eine zurückhaltende Flüssigkeitsgabe zu empfehlen. Nach aktueller Datenlage sind dadurch keine negativen Effekte auf die Nierenfunktion zu befürchten. Alternativ kann die Gabe von Humanserumalbumin erwogen werden. 
Künstliche Kolloide wie Hydroxyethylstärke werden wegen der Gefahr von Tubulusnekrosen nicht empfohlen (Patel et al. 2015).

Die Behandlung einer Koagulopathie bzw. Thrombozytopenie und Anämie erfolgt nach den Empfehlungen der aktuellen Querschnittsleitlinien der Bundesärztekammer zur Therapie mit Blutkomponenten und Plasmaderivaten (Bundesärztekammer 2020). Transfusionstrigger sind in diesem Zusammenhang nicht untersucht. Bei Fehlen klinischer Blutungszeichen kann ein Hämoglobinwert von 4,5 mmol/l (7 g/dl) bzw. bei fehlenden Zeichen einer Thrombozytenfunktionsstörung eine Thrombozytenzahl > $10 \mathrm{Gpt} / \mathrm{l}$, bei klinischen Blutungszeichen > $50 \mathrm{Gpt} / \mathrm{l}$ angestrebt werden.

Die Gerinnungsaktivierung nach Hirnverletzungen durch die Freisetzung von „tissue factor“, durch freigesetzte prokoagulatorische und proinflammatorische Proteine infolge einer Plättchenaktivierung sowie insbesondere bei traumatischen Hirnschäden durch die Freisetzung von Thromboplastin führt zu einer Koagulopathie und disseminierten intravasalen Gerinnung. Eine Fortführung der Thromboseprophylaxe ist deshalb wie bei allen Intensivpatienten sinnvoll und sollte mit niedermolekularen Heparinen erfolgen.

\subsection{Lungenfunktion}

Dorsobasale Belüftungsstörungen, bronchiale Sekretretrentionen oder Atemwegsinfektionen führen ebenfalls beim potenziellen Organspender zu sekundären pulmonalen Komplikationen und gefährden das Transplantationsergebnis. Lung-Management-Protokolle können die Zahl der erfolgreich transplantierten Organe verbessern (Miñambres et al. 2015). Das konsequente Vermeiden von Tubus- bzw. Beatmungsassoziierten Komplikationen beinhaltet die Fortführung einer lungenprotektiven Beatmung ggf. mit Recruitment-Manövern sowie die Bronchialtoilette und Lagerungsmaßnahmen. Es wird eine periphere Sauerstoffsättigung $>95 \%$ bei Normokapnie angestrebt. Die inspiratorische Sauerstoffkonzentration sollte so gering wie möglich gehalten werden. Ziel ist eine $\mathrm{PaO}_{2} / \mathrm{FiO}_{2}$-Ratio über 30o. Eine Hyperventilation mit Hypokapnie und respiratorischer Alkalose muss verhindert werden. Der Nutzen von inhalativen Beta-Sympatomimetika im Rahmen der Spenderkonditionierung ist nicht belegt (Ware et al. 2014). Durch die genannten Maßnahmen können die Zahl und Qualität der Lungentransplantationen verbessert sowie das Überleben der Transplantatempfänger verlängert werden (Angel et al. 2012; Mascia et al. 2009; Miñambres et al. 2014; Miñambres et al. 2015). 
Beispiel eines „Lung-Donor-Protocol“ (modifiziert nach Miñambres et al. 2015)

1. Hypoventilation zum Apnoe-Test ohne vorherige Dekonnektion vom Beatmungsgerät unter Beibehaltung eines positiven Atemwegsdrucks

2. Beatmung mit PEEP $8-10 \mathrm{~cm} \mathrm{H}_{2} \mathrm{O}$ und Tidalvolumina von $6-8 \mathrm{ml} / \mathrm{kg}$

3. Recruitment-Manöver stündlich und nach Dekonnektion vom Beatmungsgerät

4. Bronchoskopie mit bronchoalveolärer Lavage unmittelbar nach Hirntodfeststellung

5. Engmaschiges hämodynamisches Monitoring mit PICCO-System; Ziel: ELWI $<10 \mathrm{ml} / \mathrm{kg}$ (Flüssigkeitssubstitution, wenn erforderlich) und ZVD $<8 \mathrm{mmHg}$

6. Methylprednisolon $(15 \mathrm{mg} / \mathrm{kg}$ ) nach Hirntodfeststellung

7. Alveoläres Recruitment mit kontrollierter Beatmung (Plateau-Druck $<35 \mathrm{mmHg}$ ) mit PEEP 18-20 $\mathrm{cm} \mathrm{H}_{2} \mathrm{O}$ für 1 Minute und Reduktion um $2 \mathrm{~cm} \mathrm{H}_{2} \mathrm{O} / \mathrm{min}$; dann Steigerung des Tidalvolumens um $50 \%$ für 10 Atemzüge

8. Bei $\mathrm{PaO}_{2} / \mathrm{FIO}_{2}<300 \mathrm{mmHg}$, Halbseitenlage und Recruitment-Manöver

\section{$7.6 \quad$ Körpertemperatur}

Nach Eintreten des IHA entwickelt sich regelhaft eine Hypothermie. Sie kann neben dem vermehrten Auftreten von Arrhythmien zum Abfall der Stoffwechselleistung mit adaptiver Funktionsminderung der Organe führen. Bei zu starker Auskühlung sollte rechtzeitig eine Erwärmung über externe Wärmesysteme erfolgen. Allerdings zeigen aktuelle Studien, dass vor allem für die Nierenspende eine milde Hypothermie einen protektiven Einfluss auf die Transplantatfunktion hat (Malinoski et al. 2019; Niemann et al. 2015).

Eine milde Hypothermie sollte insbesondere vor geplanter Nierenspende zwischen $34^{\circ} \mathrm{C}$ und $35^{\circ} \mathrm{C}$ gehalten werden, scheint jedoch auch auf andere Organe ohne Nebenwirkungen.

\subsection{Hormonersatztherapie}

Der Ausfall der Hypothalamus-Hypophysen-Achse führt sowohl zu einem Abfall des Spiegels von Arginin-Vasopressin aus der Neurohypophyse und daraus resultierendem zentralen Diabetes insipidus sowie auch zu einem 
Hypocortisolismus und Hypothyreodismus durch die Beeinflussung der Adenohypophyse (Dimopoulou et al. 2003). Daraus resultieren vor allem Elektrolyt- und Flüssigkeitsstörungen sowie eine hämodynamische Instabilität (Dimopoulou et al. 2003; Mi et al. 2015; Novitzky et al. 2014; Smith 2004).

\subsubsection{Desmopressin und Arginin-Vasopressin}

Das synthetische Vasopressin-Analogon Desamino-D-Argininvasopressin (Desmopressin, DDAPV) bewirkt durch seine stärkere Wirkung an den renalen V2- als an den V1-Rezeptoren der Gefäßmuskulatur einen ausgeprägten antidiuretischen aber kaum einen vasokonstriktorischen Effekt und ist damit vor allem zur Behandlung des Diabetes insipidus centralis geeignet. Eine Polyurie mit Hypernatriämie und Hypovolämie kann mit repetitiven Einzeldosen von 1-4 $\mu \mathrm{g}$ behandelt werden.

Das Peptidhormon Arginin-Vasopressin (AVP) wirkt systemisch über die $\mathrm{V}_{\mathrm{A}^{-}}$Rezeptoren am Herzen, den glatten Gefäßmuskelzellen, den Nieren, im Myometrium, im Zentralnervensystem und der Leber, über die $V 1_{\mathrm{B}}$-Rezeptoren, die überwiegend in der Hypophyse und über die V2-Rezeptoren, die im Wesentlichen im kardiovaskulären und renalen System exprimiert werden. AVP besitzt durch seine äquipotente Affinität zu V1- und V2-Rezeptoren neben der Vasopressorwirkung ebenfalls einen therapeutischen Effekt auf den gestörten Wasser- und Elektrolythaushalt. Außerdem ist ein positiver Effekt auf die Lungenfunktion und eine signifikant bessere Organerholung bei Spendern ohne nachteilige Auswirkungen für den Empfänger verbunden. AVP sollte deshalb fester Bestandteil der Hormonersatztherapie bei der Spenderkonditionierung, vor allem aber bei Lungenspendern sein (Callahan et al. 2014; Plurad et al. 2012). Bei schweren kombinierten Störungen mit Hypotonie und Hypernatriämie/Polyurie können AVP und DDAVP parallel verabreicht werden.

\subsubsection{Kortikosteroide}

Die Kortikoidsubstitution wird nicht in erster Linie zur Behandlung der adrenocortikalen Insuffizienz eingesetzt, sondern es stehen die anti-inflammatorischen bzw. immunmodulatorischen Effekte beim Transplantatempfänger und die Reduktion des Katecholaminbedarfs zur Kreislaufstützung beim Spender im Fordergrund. Die Gabe von Methylprednisolon führte bei Lebertransplantationen zu einer Abnahme des Ischämie-Reperfusionsschadens bzw. der akuten Organ-Abstoßung (Kotsch et al. 2008). Diese Resultate konnten in einer späteren placebo-kontrollierten, randomisierten Studie bzw. in Metaanalysen zwar nicht reproduziert werden (Van Erp et al. 2018b), den- 
noch erfolgt die intravenöse Kortikosteroidgabe mit Methylprednisolon bzw. einem anderen geeigneten Kortikoid in äquivalenter Dosis routinemäßig zur Spenderpräkonditionierung, um die systemischen proinflammatorischen Effekte beim Spender zu reduzieren und die Hämodynamik zu stabilisieren.

Eine Hormonersatztherapie mit Methylprednisolon und AVP u./o. DDAVP ist in den meisten Fällen geeignet, eine kardiopulmonale und endokrine Dysregulation zu behandeln.

\subsubsection{Schilddrüsenhormone}

Eine Schilddrüsendysfunktion ist mit der Entwicklung und der Ausprägung einer Herzinsuffizienz assoziiert. Es kommt zu einer Reduktion der Kontraktilität und der Relaxation des Herzens durch Veränderungen der Myosinfasern und der Kalziumkanalfunktion, zu Bradykardien durch die Abnahme des chronotropen Effekts der Schilddrüsenhormone und durch Veränderungen an den Kaliumkanälen zu einer QT-Verlängerung mit vermehrten ventrikulären Arrhythmien (Deegan u. Furman 2011). Die Substitution von Schilddrüsenhormonen bei Organspendern und -empfängern ist nicht abschließend geklärt (Buchanan u. Mehta 2018). In retrospektiven Studien fand sich eine Zunahme der transplantierten Organe bzw. ein Überlebensvorteil bei Herztransplantierten sowohl durch die Vorbehandlung des Spenders als auch der Empfänger mit L-Thyroxin (Holndonner-Kirst et al. 2019; Novitzky et al. 2014). Eine Metaanalyse aus vier prospektiven Studien konnte dies jedoch nicht zeigen (Macdonald et al. 2012).

Während ältere Studien eine Verbesserung der Anzahl transplantierter Herzen und eine verbesserte Transplantatfunktion durch eine Hormonkombinationstherapie mit $\mathrm{T}_{3}$ bzw. T4, Methylprednisolon und Vasopressin nahelegten, waren die Ergebnisse aktuellerer randomisierter bzw. placebokontrollierter Studien nicht einheitlich (Macdonald et al. 2012; Mi et al. 2015).

Für hämodynamisch instabile Spender, die schlecht auf die herkömmlichen Therapien ansprechen bzw. im Falle einer deutlich reduzierten linksventrikulären Ejektionsfraktion $<45 \%$ ist die Gabe von Triiodthyronin oder Levothyroxin eine Therapieoption. 


\subsubsection{Hyperglykämie}

Die Blutzuckersteuerung folgt den üblichen Therapieempfehlungen in der Intensivmedizin. Angestrebt wird ein Blutzuckerspiegel im oberen Normbereich. Wenige Daten existieren speziell für die Transplantation von Pancreata. Ein niedriges Spenderalter, Normoglycämie bzw. niedrige HgbA1cSpiegel und eine Ejektionsfraktion > 50\% waren in einer kürzlich publizierten Studie unabhängige Prädiktoren für die Transplantatfunktion. Die Gabe von Insulin scheint generell unproblematisch (Sally et al. 2019).

\subsubsection{Ernährung}

Der theoretische Nutzen der Aufrechterhaltung einer Nährstoffversorgung auch nach Diagnostik des Hirntodes ergibt sich aus dem Bestreben, die Transplantatqualität zu optimieren und damit das organspezifische funktionelle Outcome beim Empfänger zu verbessern. Die enterale Ernährung wirkt der Zottenatrophie des Darms entgegen und stärkt so die mukosale Integrität des Darms. Die dadurch verringerte bakterielle Translokation wirkt sich positiv auf den Immunstatus aus und reduziert die Pneumonierate. Da die Organkonditionierung nach Abschluss der Hirntoddiagnostik noch einige Tage in Anspruch nehmen kann, kann es zu einem relevanten Defizit an Makro- und Mikronährstoffen kommen. Die infolge des erhöhten Hirndrucks häufige gastrointestinale Motilitätsstörung muss bei der enteralen Ernährung berücksichtigt werden und ggf. über eine postpylorische Ernährung unter Ableitung des Magens erfolgen.

Eine vorzugsweise enterale Ernährung sollte Bestandteil der intensivmedizinischen Maßnahmen im Rahmen der Spenderkonditionierung sein (Carrott et al. 2016).

\subsubsection{Antioxidanzien}

Im Rahmen der Reperfusion kommt es durch den Anstieg freier Sauerstoffradikale zu oxidativem Stress. Außerdem erhöht die Gabe von Röntgenkontrastmittel zur CT-Angiografie bei der IHA-Diagnostik das Risiko einer Kontrastmittelnephropathie. Die prophylaktische Gabe von antioxidativen Wirkstoffen ist ein therapeutischer Ansatz. N-Acetylcystein (NAC) hatte in einer prospektiven, randomisierten und kontrollierten Studie bei Leberspenden das Transplantatüberleben verbessert. Ein antioxidativer Effekt wurde ebenfalls für die Beatmung des Organspenders mit Sevofluran berichtet. Die Gabe von $\alpha$-Liponsäure an Spender und Empfänger bei kombinierter Nierenund Pancreas-Spende führte zur Verringerung von Entzündungsmarkern 
und reduzierte eine frühe Transplantatnephropathie und die Posttransplantat-Pankreatitis.

\section{Wegen der unzureichenden Datenlage kann keine generelle Emp- fehlung für eine Therapie mit Antioxidanzien im Rahmen der Spenderkonditionierung gegeben werden.}

\section{Tab. 3 Medikamentöse Therapieempfehlung (Auswahl)}

\begin{tabular}{|c|c|c|c|}
\hline Indikation & Substanz & $\begin{array}{l}\text { Dosierungs- } \\
\text { empfehlung }\end{array}$ & Bemerkung \\
\hline \multirow[t]{3}{*}{$\begin{array}{l}\text { Hypotonie } \\
\text { infolge erniedrigter } \\
\text { systemisch vaskulä- } \\
\text { rer Resistenz }\end{array}$} & $\begin{array}{l}\text { Arginin-Vasopressin } \\
\text { (AVP) }\end{array}$ & $0.01-0.04 \mathrm{IU} / \mathrm{min}$ & $\begin{array}{l}\text { Vasokonstriktor } \\
\text { allein oder } \\
\text { in Kombination } \\
\text { mit Noradrenalin, } \\
\text { Reduktion der } \\
\text { Noradrenalin-Dosis }\end{array}$ \\
\hline & Noradrenalin & $0,01-0,2 \mu \mathrm{g} / \mathrm{kg} / \mathrm{min}$ & $\begin{array}{l}\text { Cave! Zunahme der } \\
\text { pulmonalen Permea- } \\
\text { bilität und der koro- } \\
\text { naren/mesenterialen } \\
\text { Vasokonstriktion }\end{array}$ \\
\hline & Dopamin & $2-6 \mu \mathrm{g} / \mathrm{kg} / \mathrm{min}$ & Cave! Tachyarrhythmie \\
\hline $\begin{array}{l}\text { Hypotonie } \\
\text { infolge Links- und } \\
\text { Rechtsherzinsuffi- } \\
\text { zienz mit erhöhten } \\
\text { linksventrikulärem } \\
\text { Füllungsdruck und } \\
\text { HZV-Erniedrigung }\end{array}$ & Dobutamin & $2-5 \mu \mathrm{g} / \mathrm{kg} / \mathrm{min}$ & $\begin{array}{l}\text { Mittel der ersten Wahl } \\
\text { beim kardiogenen } \\
\text { Schock zur Frequenz- } \\
\text { steigerung (Atropin- } \\
\text { Resistenz beim Hirn- } \\
\text { funktionsverlust) }\end{array}$ \\
\hline $\begin{array}{l}\text { Diabetes insipidus } \\
\text { centralis }\end{array}$ & $\begin{array}{l}\text { Desmopressin } \\
\text { (DDAVP) }\end{array}$ & $\begin{array}{l}1-2 \mu g \text { i.v. }+2 \mu g \text { s.c., } \\
\text { ggf. Repetitionsdosen }\end{array}$ & $\begin{array}{l}\text { synthetisches Analo- } \\
\text { gon des antidiureti- } \\
\text { schen Hormons (ADH) }\end{array}$ \\
\hline $\begin{array}{l}\text { Reduktion des Kate- } \\
\text { cholaminbedarfs } \\
\text { Therapie der Hyper- } \\
\text { inflammation }\end{array}$ & Methylprednisolon & $\begin{array}{l}250 \mathrm{mg} \text { Bolus, } \\
100 \mathrm{mg} / \mathrm{h}\end{array}$ & $\begin{array}{l}\text { nach Feststellung } \\
\text { des irreversiblen } \\
\text { Hirnfunktionsverlustes }\end{array}$ \\
\hline $\begin{array}{l}\text { Therapierefraktäre } \\
\text { Herzinsuffizienz }\end{array}$ & L-Thyroxin & $\begin{array}{l}20 \mu \mathrm{g} \text { i.v. als Bolus } \\
\text { gefolgt von } 10 \mu \mathrm{g} / \mathrm{h}\end{array}$ & Therapieversuch \\
\hline Hyperglykämie & Insulin & $1-10$ I.E./h & Ziel: 4-10 mmol/l \\
\hline
\end{tabular}




\title{
Zielparameter der Kreislaufsteuerung
}

\author{
Basis-Zielparameter \\ - Normotonie (MAP > $60 \mathrm{mmHg}$, ZVD 7-10 mm Hg) \\ - Herzfrequenz $<100 / \mathrm{min}$ \\ - Euvolämie \\ - Diurese $1-2 \mathrm{ml} / \mathrm{kgKG} / \mathrm{h}$ \\ - Normothermie bis milde hypotherm (Körperkerntemperatur $>35^{\circ} \mathrm{C}$ ) \\ - Natrium i.S. $135-155 \mathrm{mmol} / \mathrm{l}$ \\ - Kalium i.S. 3,5-5 mmol/l \\ - Blutzucker $<10 \mathrm{mmol} / \mathrm{l}$ \\ - Laktat i.S. $<3 \mathrm{mmol} / \mathrm{l}$

\section{Erweiterte hämodynamische Zielparameter} \\ - Herzindex 3,0-5,0 l/min $/ \mathrm{m}^{2}$ \\ - Schlagvolumenindex (SVI) 40-60 ml/ $\mathrm{m}^{2}$ \\ - Pulmonalarterieller Verschlussdruck (PAVD) $\leq 12 \mathrm{mmHg}$ \\ - Systemisch vaskulärer Widerstandsindex (SVRI) $=2.000 \pm 500 \mathrm{dyn} *$ \\ $\mathrm{s} * \mathrm{~cm}^{-5} * \mathrm{~m}^{-2}$ \\ - Intrathorakaler Blutvolumenindex (ITBI) $850-1.000 \mathrm{ml} / \mathrm{m}^{2}$ \\ - Extravasaler Lungenwasserindex (ELWI) $3-7 \mathrm{ml} / \mathrm{kg}$ \\ Linksventrikuläre Ejektionsfraktion (LVEF) > 45\%
}

\section{Literatur}

Amado JA, Lopezespadas F, Vazquezbarquero A et al. (1995) Blood-Levels of Cytokines in Brain-Dead Patients - Relationship with Circulating Hormones and Acute-Phase Reactants. Metab Clin Exp 44:812-816. DOI: 10.1016/0026-0495(95)90198-1

Angel LF, Levine DJ, Restrepo Ml et al. (2006) Impact of a lung transplantation donor-management protocol on lung donation and recipient outcomes. Am I Respir Crit Care Med 174:710-716. DOI: 10.1164/rccm.200603-4320C

Bednarczyk JM, Fridfinnson JA, Kumar A et al. (2017) Incorporating Dynamic Assessment of Fluid Responsiveness Into Goal-Directed Therapy: A Systematic Review and Meta-Analysis. Critical Care Medicine 45:1538-1545. DOI: 10.1097/CCM.0000000000002554

Benck U, Hoeger S, Brinkkoetter PT et al. (2011) Effects of donor pre-treatment with dopamine on survival after heart transplantation: a cohort study of heart transplant recipients nested in a randomized controlled multicenter trial. Journal of the American College of Cardiology 58:1768-1777. DOI: 10.1016/j.jacc.2011.05.060

Benck U, Jung M, Krüger B et al. (2018) Donor Dopamine Does Not Affect Liver Graft Survival: Evidence of Safety From a Randomized Controlled Trial. Liver Transpl 24:1336-1345. D0I: 10.1002/ lt.25301

Birtan D, Arslantas MK, Altun GT et al. (2018) Effect of Vasoactive Therapy Used for Brain-Dead Donors on Graft Survival After Kidney Transplantation. Transplant Proc 50:1289-1291. DOI: 10.1016/j. transproceed.2018.02.058 
Borbely XI, Krishnamoorthy V, Modi S et al. (2015) Temporal Changes in Left Ventricular Systolic Function and Use of Echocardiography in Adult Heart Donors. Neurocrit Care 23:66-71. D0I: 10.1007/ s12028-014-0101-x

Buchanan IA, Mehta VA (2018) Thyroid hormone resuscitation after brain death in potential organ donors: A primer for neurocritical care providers and narrative review of the literature. Clin Neurol Neurosurg 165:96-102. D0I: 10.1016/j.clineuro.2018.01.004

Bundesärztekammer (2020) Querschnitts-Leitlinien zur Therapie mit Blutkomponenten und Plasmaderivaten - Gesamtnovelle 2020. 1-289

Bundesärztekammer (2020) Richtlinie gemäß $§ 16$ Abs. 1 S. 1 Nr. 3 TPG zur ärztlichen Beurteilung nach $\checkmark$ 9a Abs. 2 Nr. 1 TPG (RL BÄK Spendererkennung). Deutsches Ärzteblatt Ig. 117: Heft 35-36 DOI: 10.3238/arztebl.2020.rili_baek_spendererkennung_2020

Callahan DS, Neville A, Bricker S et al. (2014) The effect of arginine vasopressin on organ donor procurement and lung function. / Surg Res 186:452-457. DOI: 10.1016/j.jss.2013.09.028

Carrott P, Cherry-Bukowiec IR, Jones CM et al. (2016) Nutrition Therapy in the Organ Donor: Theoretical Benefits and Barriers to Implementation. Curr Nutr Rep 5:199-203. DOI: 10.1007/s13668-0160170-x

Citerio G, Cypel M, Dobb G) et al. (2016) Organ donation in adults: a critical care perspective. Intensive Care Med 42:305-315. DOI: 10.1007/s00134-015-4191-5

Deegan RJ, Furman WR (2011) Cardiovascular manifestations of endocrine dysfunction. I Cardiothorac Vasc Anesth 25:705-720. DOI: 10.1053/j.jvca.2010.12.001

Dimopoulou I, Tsagarakis S, Anthi A et al. (2003) High prevalence of decreased cortisol reserve in brain-dead potential organ donors. Critical Care Medicine 31:1113-1117. DOI: 10.1097/01. CCM.0000059644.54819.67

DSO (2016) Organprotektive Intensivmaßnahmen. 1-14. URL: https://www.dso.de/Leitfaden\%20fr\%20 die\%200rganspende/06.\%200rganprotektive\%20Intensivma\%C3\%9Fnahmen/DS0\%20Leitfaden\%20Kapitel\%2006.pdf (aufgerufen am 07.07.2021)

Holndonner-Kirst E, Nagy A, Czobor NR et al. (2019) The Impact of l-Thyroxine Treatment of Donors and Recipients on Postoperative Outcomes After Heart Transplantation. I Cardiothorac Vasc Anesth 33:1629-1635. DOI: 10.1053/j.jvca.2018.10.024

Kotloff RM, Blosser S, Fulda Gl et al. (2015) Management of the Potential Organ Donor in the ICU: Society of Critical Care Medicine/American College of Chest Physicians/Association of Organ Procurement Organizations Consensus Statement. Critical Care Medicine 43:1291-1325. DOI: 10.1097/ CCM. 0000000000000958

Kotsch K, Ulrich F, Reutzel-Selke A et al. (2008) Methylprednisolone therapy in deceased donors reduces inflammation in the donor liver and improves outcome after liver transplantation: a prospective randomized controlled trial. Ann Surg 248:1042-1050. DOI: 10.1097/SLA.0b013e318190e70C

Macdonald PS, Aneman A, Bhonagiri D et al. (2012) A systematic review and meta-analysis of clinical trials of thyroid hormone administration to brain dead potential organ donors. Critical Care Medicine 40:1635-1644. DOI: 10.1097/CCM.ob013e3182416ee7

Madan S, Saeed O, Vlismas P et al. (2017) Outcomes After Transplantation of Donor Hearts With Improving Left Ventricular Systolic Dysfunction. Journal of the American College of Cardiology 70:12481258. DOI: 10.1016/j.jacc.2017.07.728

Malinoski D, Patel MS, Axelrod DA et al. (2019) Therapeutic Hypothermia in Organ Donors: Follow-up and Safety Analysis. Transplantation 103:e365-e368. DOI: 10.1097/TP.0000000000002890

Malinoski DJ, Patel MS, Ahmed 0 et al. (2013) The impact of meeting donor management goals on the development of delayed graft function in kidney transplant recipients. Am J Transplant 13:9931000. DOI: 10.1111/ajt.12090 
Martin-Loeches I, Sandiumenge A, Charpentier I et al. (2019) Management of donation after brain death (DBD) in the ICU: the potential donor is identified, what's next? Intensive Care Med 45:322330. DOI: $10.1007 /$ s00134-019-05574-5

Mascia L, Mastromauro I, Viberti S et al. (2009) Management to optimize organ procurement in brain dead donors. Minerva Anestesiol 75:125-133

Mi Z, Novitzky D, Collins JF, Cooper DK (2015) The optimal hormonal replacement modality selection for multiple organ procurement from brain-dead organ donors. CLEP 7:17-27. DOI: 10.2147/CLEP. S71403

Miñambres E, Coll E, Duerto / et al. (2014) Effect of an intensive lung donor-management protocol on lung transplantation outcomes. I Heart Lung Transplant 33:178-184. DOI: 10.1016/j. healun.2013.10.034

Miñambres E, Pérez-Villares JM, Chico-Fernández M et al. (2015) Lung donor treatment protocol in brain dead-donors: A multicenter study. I Heart Lung Transplant 34:773-780. DOI: 10.1016/j. healun.2014.09.024

Nasr DM, Tomasini S, Prasad A, Rabinstein AA (2017) Acute Brain Diseases as Triggers for Stress Cardiomyopathy: Clinical Characteristics and Outcomes. Neurocrit Care 27:356-361. DOI: 10.1007/ s12028-017-0412-9

Niemann CU, Feiner J, Swain S et al. (2015) Therapeutic Hypothermia in Deceased Organ Donors and Kidney-Graft Function. N Engl I Med 373:405-414. DOI: 10.1056/NEJMoa1501969

Novitzky D, Mi Z, Sun Q et al. (2014) Thyroid hormone therapy in the management of 63,593 braindead organ donors: a retrospective analysis. Transplantation 98:1119-1127. DOI: 10.1097/ TP.0000000000000187

Patel MS, La Cruz De S, Sally MB et al. (2017) Active Donor Management During the Hospital Phase of Care Is Associated with More Organs Transplanted per Donor. Journal of the American College of Surgeons 225:525-531. DOI: 10.1016/j.jamcollsurg.2017.06.014

Patel MS, Niemann CU, Sally MB et al. (2015) The Impact of Hydroxyethyl Starch Use in Deceased Organ Donors on the Development of Delayed Graft Function in Kidney Transplant Recipients: A Propensity-Adjusted Analysis. Am I Transplant 15:2152-2158. Dol: 10.1111/ajt.13263

Plurad DS, Bricker S, Neville A et al. (2012) Arginine vasopressin significantly increases the rate of successful organ procurement in potential donors. Am / Surg 204:856-60- discussion 860-1. DOI: 10.1016/j.amjsurg.2012.05.011

Poppelaars F, Seelen MA (2016) Complement-mediated inflammation and injury in brain dead organ donors. Molecular Immunology 1-7. DOI: 10.1016/j.molimm.2016.11.004

Rahmel A (2019) Organ donation: Update 2019. Med Klin Intensivmed Notfmed 114:100-106. DOI: 10.1007/s00063-018-0513-z

Sally MB, Ellis MK, Hutchens M et al. (2019) Deceased organ donor factors influencing pancreatic graft transplantation and survival. Clin Transplant 33:e13571. DOI: 10.1111/ctr.13571

Schnuelle P, Drüschler K, Schmitt WH et al. (2019) Donor organ intervention before kidney transplantation: Head-to-head comparison of therapeutic hypothermia, machine perfusion, and donor dopamine pretreatment. What is the evidence? Am I Transplant 19:975-983. DOI: 10.1111/ ajt.15317

Schnuelle P, Schmitt WH, Weiss C et al. (2017) Effects of Dopamine Donor Pretreatment on Graft Survival after Kidney Transplantation: A Randomized Trial. Clin I Am Soc Nephrol 12:493-501. DOI: $10.2215 / \mathrm{CJN} .07600716$

Smith M (2004) Physiologic changes during brain stem death-lessons for management of the organ donor. The Journal of Heart and Lung Transplantation 23:S217-S222. D0I: 10.1016/j. healun.2004.06.017 
7 Intensivmedizinische Maßnahmen zur Aufrechterhaltung der Organfunktionen

Van Erp AC, Rebolledo RA, Hoeksma D et al. (2018a) Organ-specific responses during brain death: increased aerobic metabolism in the liver and anaerobic metabolism with decreased perfusion in the kidneys. Sci Rep 8:4405-13. DOI: 10.1038/s41598-018-22689-9

Van Erp AC, van Dullemen LFA, Ploeg RJ et al. (2018b) Systematic review on the treatment of deceased organ donors. Transplantation Reviews 32:194-206. DOI: 10.1016/j.trre.2018.06.001

Ware LB, Landeck M, Koyama T et al. (2014) A randomized trial of the effects of nebulized albuterol on pulmonary edema in brain-dead organ donors. Am I Transplant 14:621-628. DOI: 10.1111/ ajt.12564 